\title{
A Passivity-Based Approach to Voltage Stabilization in DC Microgrids*
}

\author{
Raffaele Soloperto $^{1}$, Pulkit Nahata ${ }^{1}$, Michele Tucci ${ }^{2}$, and Giancarlo Ferrari-Trecate ${ }^{1}$
}

\begin{abstract}
We consider the application of passivity theory to the problem of voltage stabilization in DC microgrids (DCmGs) given by the interconnection of Distributed Generation Units (DGUs), dynamic RL lines, and loads. We first provide a novel result on stable interconnection of multiple passive systems and then apply it to DCmGs. More specifically, we consider the decentralized controllers proposed in [1] synthesized by solving linear matrix inequalities, which allow addition and removal of DGUs in a plug-n-play fashion. We show that they passivate DGUs and combine this property with the inherent passivity of RL lines for proving voltage stability without Quasi-StationaryLine approximations, which are assumed in [1]. Theoretical results are backed up by simulations in PSCAD.
\end{abstract}

\section{INTRODUCTION}

Passivity theory provides one of the most powerful tools for the analysis of complex systems. It presents a framework for designing control actions based on considerations related to the energy of the system, both in linear and nonlinear cases. Furthermore, passivity theory has strong relations with Lyapunov stability [2]. We refer the reader to [3], [4], and the references therein, for a detailed discussion about stabilization of nonlinear systems using passivity-based approaches. For the analysis of large-scale systems, passivity provides a compositional framework, that is, passivity of a system can be shown from the passivity of its components and the way they are interconnected.

A classic result is that the feedback or parallel interconnection of two passive systems is still passive [5], [6], [7]. Compositional arguments have been also provided for stability analysis of complex interconnected systems [7], [8]. In [7], results about $\mathcal{L}_{2}$-finite-gain stability of interconected passive systems are provided. However, the interconnections must fulfill structural constraints so as to satisfy suitable Riccati inequalities. A more recent reference highlighting advantages of passivity in networked systems can be found in [8], where stability and output synchronization is shown for subsystems interconnected in a Laplacian fashion. Furthermore, [9], [10] explore passivity-based control in network of dynamical systems, in which subsystems are connected through dynamic diffusive couplings.

The primary focus of this article is the application of passivity-based tools to DC microgrids (DCmGs). DCmGs,

\footnotetext{
*This work has received support from the Swiss National Science Foundation under the COFLEX project (grant number 200021_169906).

${ }^{1}$ Raffaele Soloperto, Pulkit Nahata, and Giancarlo FerrariTrecate are with the Automatic Control Laboratory, École Polytechnique Fédérale de Lausanne (EPFL), Switzerland. Email addresses: \{raffaele.soloperto, pulkit.nahata, giancarlo.ferraritrecate\} depfl.ch

${ }^{2}$ Michele Tucci is with Dipartimento di Ingegneria Industriale e dell'Informazione, Università degli Studi di Pavia, Italy. Email address: michele.tucci02@universitadipavia.it
}

due to higher efficiency, more natural interface to many types of renewable energy sources and storge systems, and better compliance with consumer electronics, have gained traction in recent times [11], [12], [13], [14]. A key challenge in islanded DCmGs is to ensure voltage stability through decentralized control of each DGU [15]. Droop-based voltage stabilization is a commonly used decentralized approach but is plagued by load-dependent voltage deviations, propagation of voltage errors along resistive transmission lines, and presence of steady state voltage drifts [16], [15]. Plug-n-Play $(\mathrm{PnP})$ control is an another popular decentralized control strategy and allows addition or removal of DGUs with minimal human intervention [17]. Primary controllers with PnP features have been proposed in [18], [1], [16]. These regulators, however, are designed under Quasi-StationaryLine (QSL) approximation, where line inductances are neglected [19]. Such an approximation is valid for low-voltage networks with predominantly resistive lines. However, in medium-voltage and high-voltage $\mathrm{DCmGs}$, the line inductances are substantial and cannot be disregarded [20].

This paper presents a novel result on the stability of interconnected nonlinear passive systems. We consider a specific class of skew-symmetric interconnections, which are not covered by existing contributions in [7] and [8]. Moreover, the interconnections in [9], [10] are special case of the generic interconnections introduced in this work. These skew-symmetric interconnections are present in DCmGs, given as the interconnection of DGUs, loads, and dynamic RL lines, and find applications in the analysis of voltage stability. To passivate DGUs, we introduce a decentralized multivariable PI controller based on [1]. We then apply the new stability theorem to guarantee voltage stability even for arbitrarily large inductances.

The main theorem on stable interconnection of passive systems is derived in section 2 . Section 3 presents the model of DCmG, design of local voltage regulators, and the stability analysis of the closed-loop $\mathrm{mG}$. Simulations validating theoretical results are provided in Section 4. Finally, conclusions are drawn in Section 5.

\section{A. Preliminaries and notation}

1) Algebraic graph theory: We denote by $\mathcal{G}(\mathcal{V}, \mathcal{E}, \mathcal{W})$ a weighted digraph, where $\mathcal{V}=\{1, \cdots, N\}$ is the node set, $\mathcal{E} \subseteq(\mathcal{V} \times \mathcal{V})$ is the edge set, and $\mathcal{W}=\left\{w_{i j} \in \mathbb{R},(i, j) \in \mathcal{E}\right\}$ is the set of weights. If for all $(i, j) \in \mathcal{E}$, one has $(j, i) \in \mathcal{E}$, then the graph is said to be undirected, otherwise, directed. For node $i \in \mathcal{V}, \mathcal{N}_{i}^{+}=\{j \in \mathcal{V}:(i, j) \in \mathcal{E}\}$ denotes the set of out-neighbors, $\mathcal{N}_{i}^{-}=\{j \in \mathcal{V}:(j, i) \in \mathcal{E}\}$ the set of in-neighbors, and $\mathcal{N}_{i}=\mathcal{N}_{i}^{+} \cup \mathcal{N}_{i}^{-}$the set of neighbors. The 
adjacency matrix $A \in \mathbb{R}^{N \times N}$ is defined by

$$
A_{i j}=\left\{\begin{array}{ll}
w_{i j} & \text { if } j \in \mathcal{N}_{i}^{+} \\
0 & \text { otherwise }
\end{array} .\right.
$$

All digraphs in this work are assumed to be without self loops, that is, $(i, i) \notin \mathcal{E}$.

2) Passivity theory: Consider a control-affine nonlinear system

$$
\Omega=\left\{\begin{array}{l}
\dot{x}=q(x, u)=f(x)+g(x) u \\
y=h(x)
\end{array},\right.
$$

where $x \in \mathbb{R}^{n_{i}}, y \in \mathbb{R}^{1}$, and $u \in \mathbb{R}^{1}$. The functions $q$ : $\mathbb{R}^{n} \times \mathbb{R}^{p} \rightarrow \mathbb{R}^{n}, f: \mathbb{R}^{n} \rightarrow \mathbb{R}^{n}, g: \mathbb{R}^{n} \rightarrow \mathbb{R}^{n} \times \mathbb{R}^{p}$, and $h: \mathbb{R}^{n} \rightarrow \mathbb{R}^{p}$ are twice continuously differentiable, verifying $q(0,0)=0, f(0)=0$, and $h(0)=0$. Note that inputs and outputs have the same dimension $p$.

Definition 1: The nonlinear system $\Omega$ is passive [6] if there exists a continuously-differentiable positive-semidefinte storage function $\mathbf{V}: \mathbb{R}^{n} \rightarrow \mathbb{R}, \mathbf{V}(x) \geq 0, \mathbf{V}(0)=0$, and a function $\mathbf{S}: \mathbb{R}^{n} \rightarrow \mathbb{R}, \mathbf{S}(x) \geq 0 \forall t \geq 0$, such that

$$
\dot{\mathbf{V}}(x) \leq u^{T} y-\mathbf{S}(x) .
$$

The system $\Omega$ is strictly passive if $x \neq 0 \Rightarrow \mathbf{S}(x)>0$.

\section{Interconnection of Multiple Passive Systems}

Consider a set of $N$ subsystems with control-affine dynamics

$$
\begin{aligned}
\dot{x}_{i} & =f_{i}\left(x_{i}\right)+g_{i}\left(x_{i}\right) u_{i}, \\
y_{i} & =h_{i}\left(x_{i}\right)
\end{aligned}
$$

where $x_{i} \in \mathbb{R}^{n}, u_{i} \in \mathbb{R}^{p}$, and $y_{i} \in \mathbb{R}^{p}$. For modeling the interconnections between the subsystems, we introduce a weighted digraph $\mathcal{G}(\mathcal{V}, \mathcal{E}, \mathcal{W})$ where each node represents a subsystem. Suppose that the subsystems are coupled together through the input

$$
u_{i}=\sum_{j \in \mathcal{N}_{i}^{+}} w_{i j} y_{j}-\sum_{j \in \mathcal{N}_{i}^{-}} w_{j i} y_{j} \quad i=1, \cdots, N,
$$

where $w_{i j}$ are scalars. In this article, we focus on these interconnection structures. Let $A$ be the adjacency matrix associated with graph $\mathcal{G}$. We define the skew-symmetric interconnection matrix as

$$
\Phi=A-A^{T} .
$$

From (3), it is easy to verify that

$$
u=\Phi y,
$$

where $u=\left[u_{1}, \cdots, u_{N}\right]^{T}$ and $y=\left[y_{1}, \cdots, y_{N}\right]^{T}$ are the vectors of inputs and outputs respectively.

Definition 2: The interconnection between various subsystems defined by (3) is called skew symmetric.

Skew-symmetric interactions are found in electrical networks. In particular, this is the case in DC microgrids with heterogeneous components, which will be discussed further in Section III. We will now present a key property of subsystems interconnected in a skew-symmetric fashion, that is, if all the subsystems are passive, then each state $x_{i}$ converges to the zero-level set of function $\mathbf{S}_{i}\left(x_{i}\right), i \in \mathcal{V}$.

Theorem 1: Consider a set of dynamical subsystems defined by (2) coupled with each other through input (3) such that the interconnection is skew symmetric. If each subsystem is input-output passive with a radially unbounded positive-definite storage function $\mathbf{V}_{i}\left(x_{i}\right) \forall i \in \mathcal{V}$, then, for all initial conditions $x_{i}(0) \in \mathbb{R}^{n_{i}}$, the state $x=\left[x_{1}^{T}, \cdots, x_{N}^{T}\right]^{T}$ converges, as $t \rightarrow \infty$, to the largest invariant set contained in

$$
E=\left\{x=\left[x_{1}^{T} \ldots x_{N}^{T}\right]^{T}: \mathbf{S}_{i}\left(x_{i}\right)=0, i \in \mathcal{V}\right\} .
$$

Moreover, if all subsystems are strictly passive, then the origin of (2)-(3) is a globally asymptotically stable equilibrium.

Proof: The reader is deferred to [21] for a complete proof.

\section{APPLICATION OF PASSIVITY THEORY TO MGs}

In this section, we describe the electric model of a DCmG comprising of multiple DGUs connected to each other via power lines. In particular, we adopt the model in [18] which allows for general DCmG topologies.

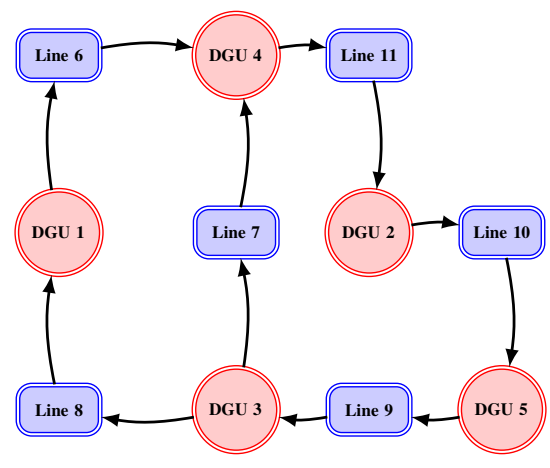

Fig. 1: A representative diagram of the DCmG network.

DC Microgrid Model: The electric interconnections in a DCmG are modeled as a directed connected graph $\mathcal{G}=$ $(\mathcal{V}, \mathcal{E}) . \mathcal{V}$ is partitioned into two sets: $\mathcal{D}=\{1, \ldots, N\}$ represents the DGUs and $\mathcal{L}=\{N+1, \cdots, M+N\}$ is the set of power lines. Each DGU is interfaced with the DCmG through a point of common coupling (PCC). For simplicity, it is assumed that the loads are connected to the DGU terminals. Indeed, even if load buses are located elsewhere, they can be mapped to PCC using Kron reduction [22]. We refer the reader to Figure 1 for a representative DCmG diagram. Since each DGU is directly connected only to the lines, all edges in $\mathcal{E}$ have one node in $\mathcal{D}$ and other in $\mathcal{L}$, making $\mathcal{G}$ a bipartite graph [23]. The orientation of each edge represents the reference direction of positive currents which is arbitrarily assigned. It is evident that a line cannot have only in-neighbors or out-neighbors as the current entering a line must leave it. Indeed, each node in $\mathcal{L}$ is always connected to two different nodes in $\mathcal{D}$ through two directed edges. We define a matrix $B \in \mathbb{R}^{N \times M}$, with DGUs along rows and 
lines along columns, as

$$
B_{i l}:\left\{\begin{array}{rl}
1 & l \in \mathcal{N}_{i}^{+} \\
-1 & l \in \mathcal{N}_{i}^{-} \text {or } l \in \mathcal{N}_{i, r}^{+}, i \in \mathcal{D}, l \in \mathcal{N} . \\
0 & \text { otherwise }
\end{array}\right.
$$

Dynamic model of power line: The power lines are represented by the $\pi$-equivalent model of the transmission line [24]. The electric scheme of the $l^{\text {th }}$ power line connecting DGUs $i$ and $j$ is shown in Figure 2. By applying Krichoff's voltage law (KVL) on the $l^{\text {th }}$ power-line, one obtains

$$
\hat{\Sigma}_{[l]}^{\text {Line }}:\left\{\frac{d I_{l}}{d t}=-\frac{R_{l}}{L_{l}} I_{l}+\frac{1}{L_{l}} \sum_{i \in \mathcal{N}_{l}} B_{i l} V_{i},\right.
$$

where the variables $V_{i}$ and $I_{l}$ represent the voltage at $P C C_{i}$ and the current flowing through the $l^{t h}$ power line respectively.

Dynamic model of the DGU: The DGU comprises a DC voltage source (usually generated by a renewable resource), a Buck converter, and a series $R L$ filter. The $i^{\text {th }}$ DGU feeds a local load at $P C C_{i}$ and is connected to other DGUs through power lines. A schematic electric diagram of the $i^{\text {th }}$ DGU along with load, connecting line(s), loads, and local PnP voltage controller is represented in Figure 2. On applying $\mathrm{KCL}$ and $\mathrm{KVL}$ on the DGU side at $P C C_{i}$, we obtain

$$
\Sigma_{[i]}^{D G U}:\left\{\begin{array}{l}
C_{t i} \frac{d V_{i}}{d t}=I_{t i}-I_{L i}-I_{i}^{*} \\
L_{t i} \frac{d I_{t i}}{d t}=-V_{i}-R_{t i} I_{t i}+V_{t i}
\end{array}, i \in \mathcal{D},\right.
$$

where $I_{i}^{*}$, a function of line currents, is the net-current injected into the $\mathrm{mG}$ and is given by

$$
I_{i}^{*}=\sum_{l \in \mathcal{N}_{i}^{+}} B_{i l} I_{l}+\sum_{l \in \mathcal{N}_{i}^{-}} B_{i l} I_{l}=\sum_{l \in \mathcal{N}_{i}} B_{i l} I_{l}, l \in \mathcal{L} .
$$

In (9), $I_{L i}$ is the load current, $V_{t i}$ is the command to the Buck converter, and $I_{t i}$ is the filter current. The terms $R_{t i} \in$ $\mathbb{R}_{>0}, L_{t i} \in \mathbb{R}_{>0}$, and $C_{t i} \in \mathbb{R}_{>0}$ are the internal resistance, inductance, and capacitance of the DGU converter.

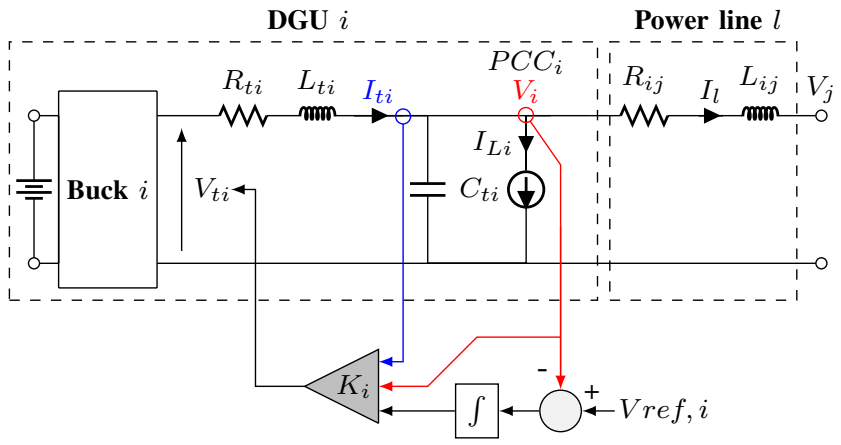

Fig. 2: Electric Scheme of $i^{\text {th }}$ DGU along with load, connecting line(s), and local PnP voltage controller.

\section{A. Design of local voltage controllers}

The main objective of local controllers is to ensure that the voltage at $P C C_{i}$ tracks a reference voltage $V_{r e f, i}$ usually provided by a higher-level controller. If the voltages are not stabilized, they can increase beyond a critical level, resulting in damage to the connected loads. A necessary condition to track a reference voltage is to steer the error $e_{[i]}(t)=$ $V_{\text {ref,i } i}(t)-V_{i}(t)$ to zero as $t \rightarrow \infty$. For this purpose, as in [1], we augment each DGU with an integrator

$$
\frac{d v_{i}}{d t}=e_{[i]}(t)=V_{r e f, i}(t)-V_{i}(t),
$$

and subsequently equip it with a state-feedback controller

$$
\mathcal{C}_{[i]}: \quad V_{t i}(t)=K_{[i]} \hat{x}_{[i]}(t),
$$

where $\hat{x}_{[i]}=\left[\begin{array}{lll}V_{i} & I_{t i} & v_{i}\end{array}\right]^{T} \in \mathbb{R}^{3}$ is the state of augmented DGU and $K_{i}=\left[k_{1, i} k_{2, i} k_{3, i}\right] \in \mathbb{R}^{1 \times 3}$ is the feedback gain. It turns out that, together with the integral action (11), controllers $\mathcal{C}_{[i]}$ define a multivariable PI regulator (see Figure 2). From (9)-(12), the closed-loop DGU model is obtained as

$$
\hat{\Sigma}_{[i]}^{D G U}:\left\{\begin{array}{l}
\frac{d V_{i}}{d t}=\frac{1}{C_{t i}} I_{t i}-\frac{1}{C_{t i}} I_{L i}-\frac{1}{C_{t i}} I_{i}^{*} \\
\frac{d I_{t i}}{d t}=\alpha_{i} V_{i}+\beta_{i} I_{t i}+\gamma_{i} v_{i} \\
\frac{d v_{i}}{d t}=-V_{i}+V_{\text {ref }, i}
\end{array},\right.
$$

where $\alpha_{i}=\frac{\left(k_{i, 1}-1\right)}{L_{t i}}, \beta_{i}=\frac{\left(k_{i, 2}-R_{t i}\right)}{L_{t i}}$, and $\gamma_{i}=\frac{k_{i, 3}}{L_{t i}}$. In particular, the control architecture is decentralized since the computation of $V_{t i}$ requires the state of $\hat{\Sigma}_{[i]}^{D G U}$ only. It is important to highlight that, in general, decentralized design of local regulators can fail to guarantee voltage stability of the whole $\mathrm{mG}$ [18], [1]. This is due to the fact that DGUs interact with grid through $I_{i}^{*}$ which in turn is a function of PCC voltages and line currents.

\section{B. Stability of the $m G$}

When DGUs are equipped with controllers (12), the whole $\mathrm{mG}$ can be stabilized. We will exploit skew-symmetric interactions and passivity to guarantee the stability of the origin of DCmG described by (8), (10), and (13). The system (13) can be equivalently written as

$$
\hat{\Sigma}_{[i]}^{D G U}: \dot{\hat{x}}_{[i]}=\hat{f}_{[i]}\left(\hat{x}_{[i]}\right)+\hat{g}_{[i]}\left(\hat{x}_{[i]}\right) \hat{u}_{[i]}+\varphi_{[i]},
$$

where $\hat{f}_{[i]}\left(\hat{x}_{[i]}\right)=\hat{A}_{[i]} \hat{x}_{[i]} \in \mathbb{R}^{3}, \hat{g}_{[i]}\left(\hat{x}_{[i]}\right)=\hat{B}_{[i]} \in \mathbb{R}^{3}$, $\varphi_{[i]}=\left[\begin{array}{lll}-C_{t i}^{-1} \bar{I}_{L i} & 0 & V_{\text {ref }, i}\end{array}\right]^{T} \in \mathbb{R}^{3}, \hat{y}_{[i]}=\hat{h}_{[i]}\left(\hat{x}_{[i]}\right)=V_{i}$, and $\hat{u}_{[i]}=-I_{i}^{*}$. Matrices $\hat{A}_{[i]}, \hat{B}_{[i]}$ are defined as

$$
\hat{A}_{[i]}=\left[\begin{array}{ccc}
0 & \frac{1}{C_{t i}} & 0 \\
\alpha_{i} & \beta_{i} & \gamma_{i} \\
-1 & 0 & 0
\end{array}\right], \text { and } \hat{B}_{[i]}=\left[\begin{array}{c}
\frac{1}{C_{t i}} \\
0 \\
0
\end{array}\right]
$$

Similarly, from (8), one has

$$
\hat{\Sigma}_{[l]}^{\text {Line }}: \dot{\hat{x}}_{[l]}=\hat{f}_{[l]}\left(\hat{x}_{[l]}\right)+\hat{g}_{[l]}\left(\hat{x}_{[l]}\right) \hat{u}_{[l]},
$$


where $\hat{x}_{[l]}=I_{l}, \hat{f}_{[l]}\left(\hat{x}_{[l]}\right)=\frac{R_{l}}{L_{l}} I_{l}, \hat{g}_{[l]}\left(\hat{x}_{[l]}\right)=\frac{1}{L_{l}}, \hat{y}_{[l]}=$ $\hat{h}_{[l]}\left(\hat{x}_{[l]}\right)=I_{l}$, and $\hat{u}_{[l]}=\sum_{i \in \mathcal{N}_{l}} B_{i l} V_{i}$.

Our main aim is to apply Theorem 1 which requires control-affine dynamics defined in (2). The vector $\varphi_{[i]}$ in (14), varying for different choices of constant exogenous inputs $V_{\text {ref }, i}$ and $\bar{I}_{L i}$, must be removed to match the form in (2). Note that equations (14) and (15), defining the closedloop dynamics of the $\mathrm{DCmG}$, are linear. Therefore, the stability of equilibrium in the absence of $\varphi_{[i]}$ is equivalent to the stability of all the equilibria generated for different (yet constant) $\varphi_{[i]}$. One obtains the DCmG dynamics as

$$
\begin{aligned}
\hat{\Sigma}_{[i]}^{D G U}: & : \dot{\hat{x}}_{[i]}=\hat{f}_{[i]}\left(\hat{x}_{[i]}\right)+\hat{g}_{[i]}\left(\hat{x}_{[i]}\right) \hat{u}_{[i]} \\
\hat{\Sigma}_{[l]}^{\text {Line }}: & : \dot{\hat{x}}_{[l]}=\hat{f}_{[l]}\left(\hat{x}_{[l]}\right)+\hat{g}_{[l]}\left(\hat{x}_{[l]}\right) \hat{u}_{[l]}, i \in \mathcal{D}, l \in \mathcal{L} .
\end{aligned}
$$

We are now in a position to apply Theorem 1 . The next lemma, in essence, shows that if one can prove passivity of lines and closed-loop DGUs, then one can localize the asymptotic behavior of the states.

Lemma 1: Under the assumption that there exist positivedefinite storage functions $\widehat{\mathbf{V}}_{[i]}\left(\hat{x}_{[i]}\right)$ and $\widehat{\mathbf{V}}_{[l]}\left(\hat{x}_{[l]}\right)$ for all $i \in$ $\mathcal{D}, l \in \mathcal{L}$ such that $\hat{\Sigma}_{[i]}^{D G U}$ and $\hat{\Sigma}_{[l]}^{L i n e}$ in (14) are passive, then the state $\hat{x}=\left[\hat{x}_{[1]}, \cdots, \hat{x}_{[N+M]}\right]^{T}$ asymptotically converges to the largest invariant set in

$$
E=\left\{\hat{x}_{[i]}, \hat{x}_{[l]}: \widehat{\mathbf{S}}_{[i]}\left(\hat{x}_{[i]}\right)=0, \widehat{\mathbf{S}}_{[l]}\left(\hat{x}_{[l]}\right)=0, i \in \mathcal{D}, l \in \mathcal{L}\right\} .
$$

Proof: The input to $\hat{\Sigma}_{[i]}^{D G U}(14)$ is

$$
\hat{u}_{[i]}=\sum_{l \in \mathcal{N}_{i}}-B_{i l} \hat{y}_{[l]}=\sum_{l \in \mathcal{N}_{i}^{+}} \underbrace{-B_{i l}}_{w_{i l}} \hat{y}_{[l]}-\sum_{l \in \mathcal{N}_{i}^{-}} \underbrace{B_{i l}}_{w_{l i}} \hat{y}_{[l]} .
$$

Using (7), one obtains

$$
w_{i l}=-1 l \in \mathcal{N}_{i}^{+} \text {and } w_{l i}=-1 l \in \mathcal{N}_{i}^{-} .
$$

Also, for line $\hat{\Sigma}_{[l]}^{\text {Line }}$

$$
\hat{u}_{[l]}=\sum_{i \in \mathcal{N}_{l}} B_{i l} \hat{y}_{[l]}=\sum_{i \in \mathcal{N}_{l}^{+}} \underbrace{B_{i l}}_{w_{l i}} \hat{y}_{[i]}-\sum_{i \in \mathcal{N}_{l}^{-}} \underbrace{-B_{i l}}_{w_{i l}} \hat{y}_{[i]} .
$$

Note that if $i \in \mathcal{N}_{l}^{+}$, then $l \in \mathcal{N}_{i}^{-}$and $B_{i l}=-1$. Conversely, $B_{i l}=1$ if $i \in \mathcal{N}_{l}^{-}$. Therefore,

$$
w_{l i}=-1 i \in \mathcal{N}_{l}^{+} \text {and } w_{i l}=-1 i \in \mathcal{N}_{l}^{-} \text {. }
$$

From (18) and (19),

$$
w_{i j}=-1, i, j \in \mathcal{D} \cup \mathcal{L}
$$

Since $\hat{u}_{[i]}$ and $\hat{u}_{[l]}$ correspond to the coupling defined in (3), the interconnection is skew symmetric with

$$
\Phi=A-A^{T}=\left[\begin{array}{cc}
\mathbf{0} & -B \\
B^{T} & \mathbf{0}
\end{array}\right] .
$$

As a direct consequence of Theorem 1, each state $\hat{x}_{[k]}$ asymptotically converges to the largest invariant set in the zero-level set $E$ of the function $\mathbf{S}_{[k]}\left(\hat{x}_{[k]}\right), k \in \mathcal{V}$.
1) Passivity of DGUs and lines: The passivity of DGUs and the power lines is essential to guarantee the stability of DCmG. As shown in [7], the power lines are strictly passive with a positive-definite storage function $\widehat{\mathbf{V}}_{[l]}\left(\hat{x}_{[l]}\right)=\frac{1}{2} L_{l} \hat{x}_{[l]}^{2}$ and $\widehat{\mathbf{S}}_{[l]}\left(\hat{x}_{[l]}\right)=R_{l} \hat{x}_{[l]}^{2}$. Given the passivity of lines, the next step is to ensure the passivity of the DGUs. This will be acheived by desigining the feedback gains $K_{[i]}$ as shown in [1] and recalled next. Similar to [1], we propose a candidate storage function of the structure

$$
\widehat{\mathbf{V}}_{[i]}\left(\hat{x}_{[i]}\right)=\frac{1}{2} \hat{x}_{[i]}^{T} \underbrace{\left[\begin{array}{cc}
C_{t i} & 0 \\
0 & \mathcal{P}_{[i]}
\end{array}\right]}_{\hat{P}_{[i]} \in \mathbb{R}^{3 \times 3}} \hat{x}_{[i]}, i \in \mathcal{D}
$$

where $\mathcal{P}_{[i]} \in \mathbb{R}^{2 \times 2}$. For $\widehat{\mathbf{V}}_{[i]}\left(\hat{x}_{[i]}\right)>0$, the matrix $\hat{P}_{[i]}$ must be positive definite. On computing the derivatives of $\widehat{\mathbf{V}}_{[i]}\left(\hat{x}_{[i]}\right)$ along the trajectory of $\hat{\Sigma}_{[i]}^{D G U}$ of (16), one obtains

$$
\begin{aligned}
\dot{\hat{\mathbf{V}}}_{[i]}\left(\hat{x}_{[i]}\right) & =\frac{1}{2} \hat{x}_{[i]}^{T} \hat{P}_{[i]} \dot{\hat{x}}_{[i]}+\frac{1}{2} \dot{\hat{x}}_{[i]}^{T} \hat{P}_{[i]} \hat{x}_{[i]} \\
& =\hat{u}_{[i]}^{T} \hat{y}_{[i]}-\underbrace{\hat{x}_{[i]}^{T} \hat{Q}_{[i]}\left(\hat{x}_{[i]}\right) \hat{x}_{[i]}}_{\widehat{\mathbf{S}}_{[i]}\left(\hat{x}_{[i]}\right)},
\end{aligned}
$$

where

$$
\hat{Q}_{[i]}=\hat{P}_{[i]} \hat{A}_{[i]}+\hat{A}_{[i]}^{T} \hat{P}_{[i]} .
$$

For $\widehat{\mathbf{S}}_{[i]}\left(\hat{x}_{[i]}\right) \geq 0$, the matrix $\hat{Q}_{[i]}$ must be positive definite or positive semidefinite.

Remark 1: By direct computation, one has $\hat{Q}_{[i, 11]}=0$, showing that $\hat{Q}_{[i]}$ cannot be positive definite, as its first minor is not positive. Hence, for the choice of storage function (21), $\hat{\Sigma}_{[i]}^{D G U}$ can never be strictly passive.

The matrix $\hat{P}_{[i]}$ must be positive definite and the gains $K_{[i]}$ should be chosen in a way such that $\hat{Q}_{[i]}$ is positive semidefinite. The problem of computing suitable $\hat{P}_{[i]}$ and $K_{[i]}$ can be cast into a linear matrix inequality and is solved using numerical optimization. We refer the reader to [1] for further details.

2) Asymptotic stability analysis: The power lines are strictly passive and the DGUs can be passivated using numerical optimization. At this point, one can invoke Lemma 1 to conclude that the states asymptotically converge to the largest invariant set in $E$ defined by (17). Furthermore, one can show that this set contains only the origin of the statespace, hence proving the following result.

Theorem 2: If $\hat{\Sigma}_{[i]}^{D G U}$ is passive for all $i \in \mathcal{D}$, then the origin of (16) is asymptotically stable.

Proof: The proof is provided in [21] and is omitted due to space constraints.

Remark 2 (PnP nature of control): From (23), for computing $K_{[i]}$ such that $\hat{Q}_{[i]}$ is positive semidefinite, one needs the knowledge of matrix $\hat{A}_{[i]}$. It should be noted that $\hat{A}_{[i]}$ depends only on the filter parameters of the $i^{t h}$ DGU. Therefore, controller $\mathcal{C}_{[i]}$ can be synthesized in a purely decentralized fashion. We defer the reader to [1] for detailed comments about the benefits of this PnP design procedure. 
Moreover, controller $\mathcal{C}_{[i]}$ is completely independent from the computation of controllers $\mathcal{C}_{[j]}, i, j \in \mathcal{D}$.

\section{Simulation RESUlts}

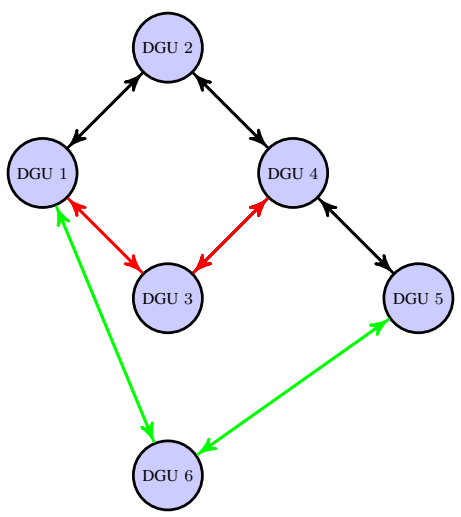

Fig. 3: Simplified representation $\mathrm{mG}$ composed of DGUs. The connecting power lines are represented by the edges, where the green and red edges, respectively, represent plugging and unplugging of a DGU.

In this section, we aim to validate the developed passivitybased framework for analyzing the stability of DCmG through simulation studies conducted in PSCAD. We consider a scenario similar to one presented in [1]: a meshed $\mathrm{mG}$ composed of 6 DGUs (see Figure 3) with non-identical electrical parameters. The reader is defered to [1] for parameter values used in the simulation. Even for simulations in [1], dynamic RL lines with very low inductances were used. However, in this setup, the line inductances have been increased tenfold to demonstrate the usefulness of the theorem. In our experiments, voltage references $V_{\text {ref, } i}$, $i=1, \ldots, 6$ to be tracked at each PCC are set to slightly different values, hence allowing current flow through power lines in the asymptotic regime.

1) Plug-in of a new DGU: At the beginning of the simulation, DGUs 1-5 are connected together while DGU 6 is isolated. At time $t=4 \mathrm{~s}$, we connect $\hat{\Sigma}_{[6]}^{D G U}$ to $\hat{\Sigma}_{[1]}^{D G U}$ and $\hat{\Sigma}_{[5]}^{D G U}$. As mentioned in Remark 2, since local regulator design hinges on parameters of the corresponding DGU only, the update of the controllers of DGUs 1, 5 and 6 is not required. In Figure 4, we notice very small deviations of the output voltages at PCCs 1, 5, and 6 from their references around the plug-in time.

2) Unplugging of a $D G U$ : At time $t=12 \mathrm{~s}$, we simulate the disconnection of $\hat{\Sigma}_{[3]}^{D G U}$. There is no need to update the controllers of DGUs neighboring $\hat{\Sigma}_{[6]}^{D G U}$ (in this case, DGUs 1 and 4). As shown in Figure 5, the voltages at PCCs 1 and 4 around the unplugging time, exhibit small deviations from the corresponding references and are promptly restored by the control actions.

\section{Conclusions}

We presented a passivity-based approach to the problem of voltage stability in DCmGs. Different from existing works
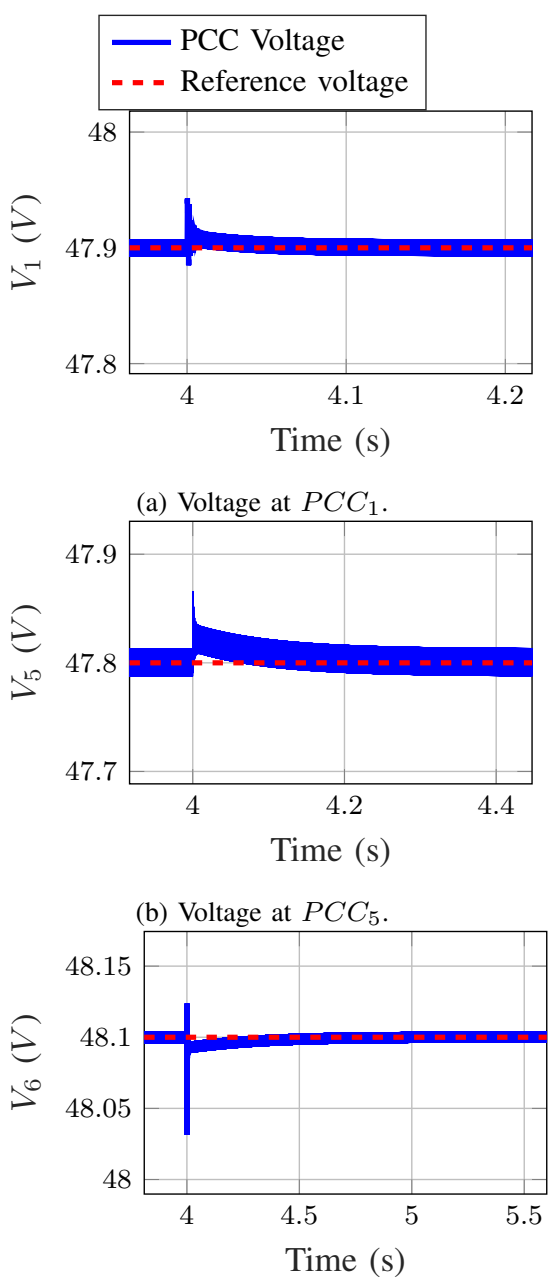

(c) Voltage at $P C C_{6}$.

Fig. 4: Performance of the implemented decentralized controllers $\mathcal{C}_{[i]}$ during the plug-in of DGU 6 at time $t=4 \mathrm{~s}$.

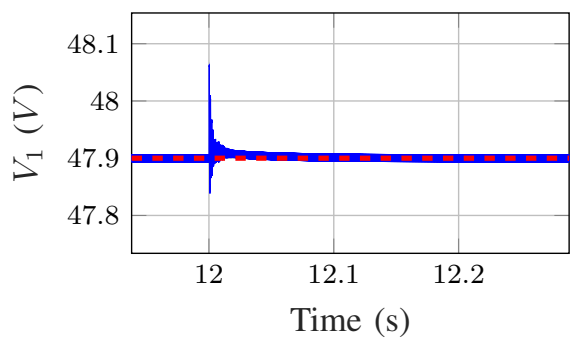

(a) Voltage at $P C C_{1}$.

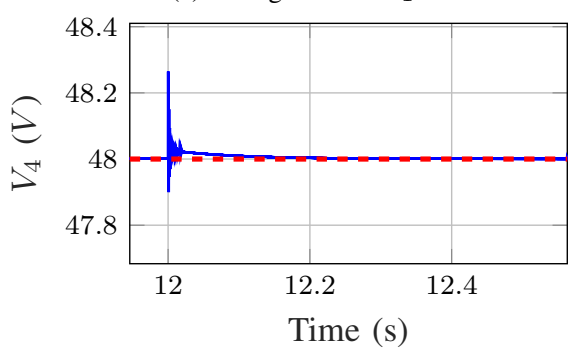

(b) Voltage at $\mathrm{PCC}_{4}$.

Fig. 5: Performance of the implemented decentralized controllers $\mathcal{C}_{[i]}$ during the unplugging of DGU 3 at $t=12 \mathrm{~s}$. 
[18], [1], [16], our $\mathrm{mG}$ model considers the dynamic nature of RL lines. The control design is fully decentralized and allows removal and addition of DGUs in a PnP fashion. Many interesting future research directions can be taken. The first one is to consider the application of proposed passivity-based framework to $\mathrm{AC} \mathrm{mGs.} \mathrm{Another} \mathrm{one} \mathrm{is} \mathrm{the} \mathrm{inclusion} \mathrm{of} \mathrm{more}$ sophisticated load models like ZIP loads. Finally, the compositional property of passivity can be exploited for design of hierarchical control scheme to achieve advanced objectives like current and power sharing and $\mathrm{mG}$ optimization.

\section{REFERENCES}

[1] M. Tucci, S. Riverso, and G. Ferrari-Trecate, "Line-independent plugand-play controllers for voltage stabilization in DC microgrids," IEEE Transactions on Control Systems Technology, vol. PP, no. 99, pp. 1-9, 2017.

[2] N. Kottenstette, M. J. McCourt, M. Xia, V. Gupta, and P. J. Antsaklis, "On relationships among passivity, positive realness, and dissipativity in linear systems," Automatica, vol. 50, no. 4, pp. 1003-1016, 2014.

[3] R. Ortega, Z. P. Jiang, and D. J. Hill, "Passivity-based control of nonlinear systems: a tutorial," in Proceedings of the 1997 American Control Conference (Cat. No.97CH36041), vol. 5, June 1997, pp. 2633-2637 vol.5.

[4] C. Ebenbauer, T. Raff, and F. Allgöwer, "Dissipation inequalities in systems theory: An introduction and recent results," in Invited Lectures of the International Congress on Industrial and Applied Mathematics, vol. 2007, 2009, pp. 23-42.

[5] C. A. Desoer and M. Vidyasagar, Feedback systems: input-output properties. SIAM, 2009.

[6] H. K. Khalil, Noninear Systems. Prentice-Hall, New Jersey, 1996.

[7] B. Brogliato, R. Lozano, B. Maschke, and O. Egeland, Dissipative Systems Analysis and Control. London: Springer, 2007.

[8] N. Chopra, "Output synchronization on strongly connected graphs," IEEE Transactions on Automatic Control, vol. 57, no. 11, pp. 2896-2901, 2012.

[9] M. Burger and C. D. Persis, "Dynamic coupling design for nonlinear output agreement and time-varying flow control," Automatica, vol. 51, pp. 210 - 222, 2015. [Online]. Available: http://www.sciencedirect. com/science/article/pii/S0005109814004622

[10] J. Xiang, Y. Li, and D. J. Hill, "Cooperative output regulation of linear multi-agent network systems with dynamic edges," Automatica, vol. 77, pp. 1 - 13, 2017. [Online]. Available: http://www.sciencedirect.com/science/article/pii/S0005109816304514

[11] T. Dragicevic, J. C. Vasquez, J. M. Guerrero, and D. Skrlec, "Advanced LVDC electrical power architectures and microgrids: A step toward a new generation of power distribution networks." IEEE Electrification Magazine, vol. 2, no. 1, pp. 54-65, March 2014.

[12] J. W. Simpson-Porco, F. Dörfler, and F. Bullo, "On resistive networks of constant-power devices," IEEE Transactions on Circuits and Systems II: Express Briefs, vol. 62, no. 8, pp. 811-815, Aug 2015.

[13] M. Karami and R. M. Cuzner, "A distributed controller for DC microgrids stability enhancement," in 2016 IEEE International Conference on Renewable Energy Research and Applications (ICRERA), Nov 2016, pp. 556-561.

[14] C. De Persis, E. Weitenberg, and F. Dörfler, "A power consensus algorithm for DC microgrids," Automatica, 2016, submitted.

[15] T. Dragicevic, X. Lu, J. C. Vasquez, and J. M. Guerrero, "DC microgrids part 1: A review of control strategies and stabilization techniques," IEEE Transactions on Power Electronics, vol. 31, no. 7, pp. 4876-4891, July 2016.

[16] J. Zhao and F. Dörfler, "Distributed control and optimization in DC microgrids," Automatica, vol. 61, pp. 18 - 26, 2015.

[17] L. Meng, Q. Shafiee, G. F. Trecate, H. Karimi, D. Fulwani, X. Lu, and J. M. Guerrero, "Review on control of DC microgrids and multiple microgrid clusters," IEEE Journal of Emerging and Selected Topics in Power Electronics, vol. 5, no. 3, pp. 928-948, Sept 2017.

[18] M. Tucci, S. Riverso, J. C. Vasquez, J. M. Guerrero, and G. FerrariTrecate, "A decentralized scalable approach to voltage control of DC islanded microgrids," IEEE Transactions on Control Systems Technology, vol. 24, no. 6, pp. 1965-1979, Nov 2016.
[19] V. Venkatasubramanian, H. Schattler, and J. Zaborszky, "Fast timevarying phasor analysis in the balanced three-phase large electric power system," IEEE Transactions on Automatic Control, vol. 40, no. 11, pp. 1975-1982, Nov 1995.

[20] M. Andreasson, E. Tegling, H. Sandberg, and K. H. Johansson, "Performance and scalability of voltage controllers in multi-terminal HVDC networks," in American Control Conference, 2017.

[21] P. Nahata, R. Soloperto, M. Tucci, A. Martinelli, and G. FerrariTrecate, "A passivity-based approach to voltage stabilization in DC microgrids with ZIP loads," Automatica, 2017, submitted.

[22] F. Dörfler and F. Bullo, "Kron reduction of graphs with application to electrical networks," IEEE Transactions on Circuits ad Systems 1: Regular papers, vol. 60(1), pp. 150 - 163, 2013.

[23] A. S. Asratian, T. Denley, and R. Häggkvist, Bipartite Graphs and Their Applications. New York, NY, USA: Cambridge University Press, 1998.

[24] P. Kundur, Power System Stability and Control. McGraw-Hill, 1994. 